Document downloaded from:

http://hdl.handle.net/10251/50821

This paper must be cited as:

Ponsoda Miralles, E.; Blanes Zamora, S. (2014). Exponential integrators for coupled selfadjoint non-autonomous partial differential systems. Applied Mathematics and Computation. 243:1-11. doi:10.1016/j.amc.2014.05.050.

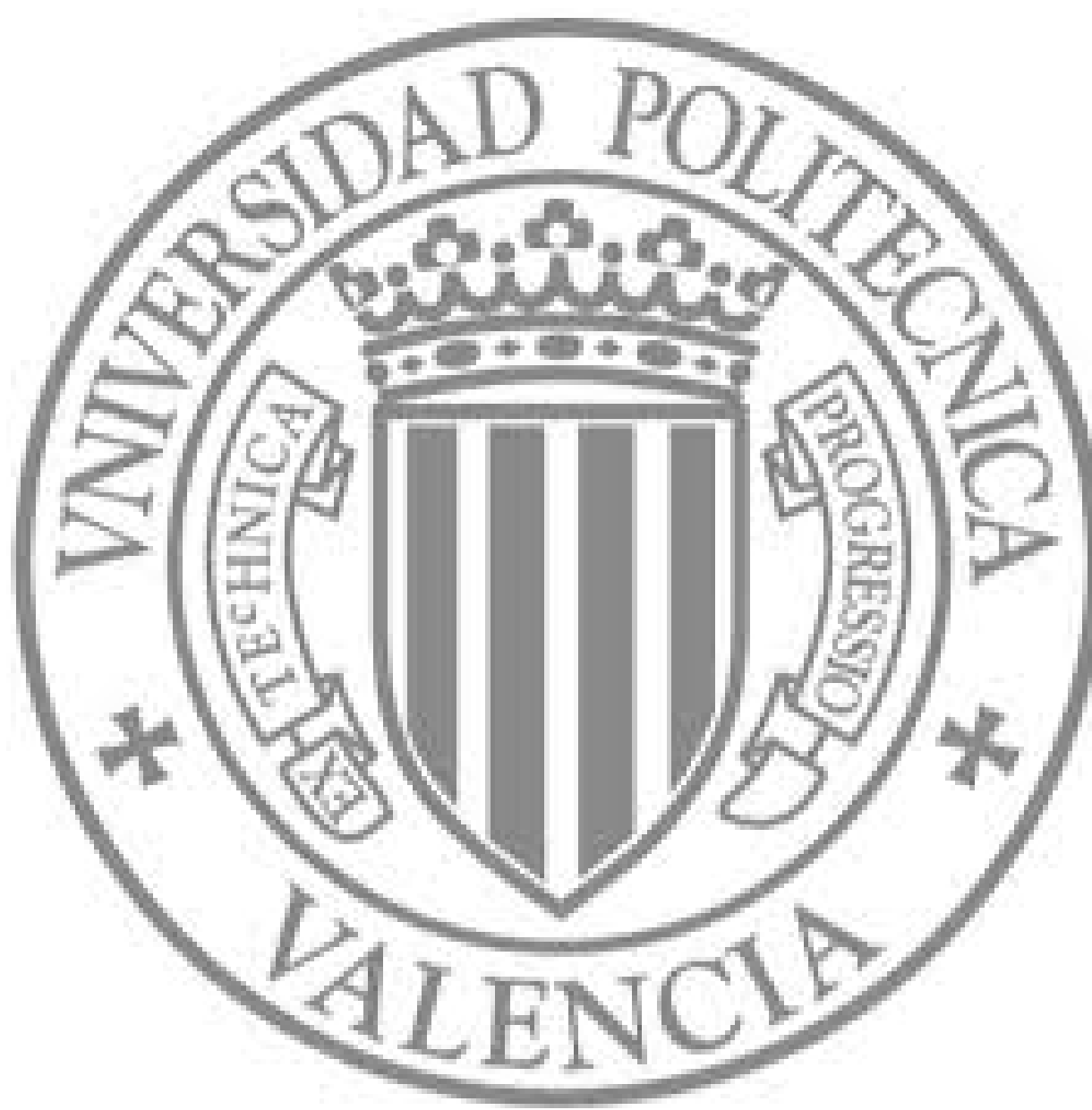

The final publication is available at

http://dx.doi.org/10.1016/j.amc.2014.05.050

Copyright Elsevier 


\title{
Exponential integrators for coupled self-adjoint non-autonomous partial differential systems
}

\author{
Enrique Ponsoda ${ }^{1}$ \& Sergio Blanes ${ }^{2}$ \\ Instituto Universitario de Matemática Multidisciplinar \\ Universitat Politècnica de València. Building $8 G$ \\ 46022 Valencia. Spain
}

\begin{abstract}
In this paper, a numerical algorithm that approximates suitably the solution of certain non-autonomous partial differential systems of equations is constructed. Firstly, conditions to be satisfied for the data of the problem to ensure the existence of a unique solution is established and, after using a matrix separation of variables technique, an exact series solution is constructed. Each term in the series solution must solve a linear system of non-autonomous differential equations with oscillatory solutions. In order to approximate numerically the formal series solution, schemes based on the Magnus expansions are considered, and we show that commutator-free Magnus integrators are of great interest for solving this problem. We show that this family of methods admit a recursive relation which provides numerical approximations for all terms in the series solution. This iterative recursion has similar error growth as the exact solution, and this ensures the convergence of the numerical solution. These exponential integrators usually often provide good results, generally better than other standard numerical methods, both from the standpoint of preserving qualitative properties of the problem and the computational cost. Finally, numerical experiments are considered in order to test the behavior of the constructed algorithm.
\end{abstract}

Keywords: Non-autonomous matrix partial differential systems, separation of variables technique, series solution, numerical approximation, Magnus expansions.

\footnotetext{
${ }^{1}$ Email address: eponsoda@imm.upv.es

${ }^{2}$ Email address: serblaza@imm.upv.es
} 


\section{Introduction}

Let us consider the numerical integration of self-adjoint partial differential equations of the type

$$
\left(P(t) u_{t}(x, t)\right)_{t}=Q(t) u_{x x}(x, t) ; \quad 0 \leq x \leq d, \quad t \geq 0,
$$

with initial and boundary conditions given by

$$
\left.\begin{array}{rl}
u(0, t)=u(d, t)=0, & t \geq 0, \\
u(x, 0)=f(x), & 0 \leq x \leq d, \\
u_{t}(x, 0)=g(x), & 0 \leq x \leq d,
\end{array}\right\}
$$

where $u(x, t) \in \mathbb{R}^{r}$. We consider the case where:

I) $P(t), Q(t) \in \mathbb{R}^{r \times r}$ are positive definite matrices

II) $-P^{\prime}(t)$ and $Q^{\prime}(t)$ are both positive (or negative) semidefinite matrices.

III) $f(x)$ is three times differentiable and $f^{(3)}(x)$ is piecewise continuous in $[0, d]$ with $f(0)=f(d)=f^{(2)}(0)=f^{(2)}(d)=0$.

IV) $g(x)$ is twice differentiable with $g^{(2)}(x)$ piecewise continuous in $[0, d]$ and $g(0)=g(d)=0$.

The system (1) appears frequently in the study of microwave heating processes, where the variations of the dielectric properties of the material with temperature, density, moisture content and other parameters make the system non-autonomous, see [10,13] for more details. Systems of type (1) can also be found on models for the study of electromagnetic processing of homogeneous materials at high power densities or analysis of multi mode microwave applicators, see $[8,17]$

Under conditions I)-IV), the problem (1)-(2) has, at most, a twice continuously differentiable solution [16, Section 2]. We look for a numerical solution, and to this purpose we first consider separation of variables. The solution can be formally written, in a bounded domain $D(d, T)=\{(x, t)$; $0 \leq x \leq d, 0 \leq t \leq T\}$, for a given $T>0$, as a convergent series

$$
u(x, t)=\sum_{n \geq 1}\left\{Y_{n}(t) a_{n}+\widetilde{Y}_{n}(t) b_{n}\right\} \sin \left(\frac{n \pi x}{d}\right),
$$


where $a_{n}, b_{n} \in \mathbb{R}^{r}$ are given by

$$
a_{n}=\frac{2}{d} \int_{0}^{d} f(x) \sin \left(\frac{n \pi x}{d}\right) d x, \quad b_{n}=\frac{2}{d} \int_{0}^{d} g(x) \sin \left(\frac{n \pi x}{d}\right) d x .
$$

The matrices $Y_{n}(t), \widetilde{Y}_{n}(t) \in \mathbb{R}^{r \times r}$ are given by

$$
Y_{n}(t)=\left[\begin{array}{ll}
I_{r} & 0_{r \times r}
\end{array}\right] V_{n}(t), \quad \widetilde{Y}_{n}(t)=\left[\begin{array}{ll}
I_{r} & 0_{r \times r}
\end{array}\right] W_{n}(t),
$$

with $V_{n}(t), W_{n}(t) \in \mathbb{R}^{2 r \times r}$ verifying the initial value problems (IVPs)

$$
\begin{array}{rlrl}
V_{n}^{\prime}(t) & =M(t, n) V_{n}(t), & V_{n}(0) & =\left[\begin{array}{c}
I_{r} \\
0_{r \times r}
\end{array}\right] ; \\
W_{n}^{\prime}(t)=M(t, n) W_{n}(t), & W_{n}(0)=\left[\begin{array}{c}
0_{r \times r} \\
P(0)
\end{array}\right] ;
\end{array}
$$

where

$$
M(t, n)=\left[\begin{array}{cc}
0_{r \times r} & P^{-1}(t) \\
-\left(\frac{n \pi}{d}\right)^{2} Q(t) & 0_{r \times r}
\end{array}\right] \in \mathbb{R}^{2 r \times 2 r},
$$

see [16] for details. Here, $0_{r \times r}, I_{r} \in \mathbb{R}^{r \times r}$ denote the null and identity matrices, respectively. Note that from our assumptions, $P(t), Q(t)$ are nonsingular matrices for all $t \geq 0$.

Given a tolerance, it is possible to find $n_{0}$ such that the truncated series for $n \leq n_{0}$ provides an approximate solution close to the exact solution bounded by the tolerance. However, the solution for the matrices $Y_{n}(t), \widetilde{Y}_{n}(t), n=$ $1,2, \ldots, n_{0}$ needs, in general, to be carried numerically (typically on a mesh $0<t_{1}<t_{2}<\ldots<t_{L}$ where $L$ also depends on $n_{0}$ ), being this the most costly part for the algorithm. Notice the matrix $M(t, n)$ in (8) has purely imaginary eigenvalues which are at the same time proportional to $n$, making the solution highly oscillatory for large values of $n$, and standard methods do not show a good performance in that case.

Implicit methods are, in general, required to numerically solve the equations. However, in general, one has that $L=\mathcal{O}\left(n_{0}^{2}\right)$, i.e. the mesh size has to be chosen inversely proportional to $n_{0}^{2}$ and, in each interval, the method has to be applied $n_{0}$ times (for $n=1,2, \ldots, n_{0}$ ). As a result, the matrices $Q(t)$ and $P^{-1}(t)$ need to be evaluated in a number of mesh points which grows as $n_{0}^{2}$, and the method has to be applied $\mathcal{O}\left(n_{0}^{3}\right)$ times. 
On the other hand, most exponential integrators can deal efficiently with the numerical solution for relatively large values of $n$. Usually, one can take $L=\mathcal{O}\left(n_{0}\right)$ and on each mesh the exponentials have to be computed for each value of $n \leq n_{0}$.

We consider, however, a class of exponential integrators based on the Magnus series expansion which provide very accurate solutions and the exponentials have to computed only for $n=1$. Then, using a simple recursive relation one can find the corresponding values for $n>1$. The methods used are referred as commutator-free Magnus integrators. The numerical solutions obtained are also such that the series solution remains still convergent.

The paper is organized as follows. In section 2 and based on section 4 of [16], the convergence of the formal series solution (3) is established. In section 3, numerical methods based on Magnus expansion are proposed in order to solve the IVPs (6)-(8). Exploiting the structure of the matrices, we found that the computational cost of the proposed method is very advantageous when is compared with other standard numerical methods. The convergence of the series obtained by the numerical scheme is studied in section 4 . Section 5 deals with the presentation of numerical experiments in order to test the effectiveness of the proposed algorithm. Conclusions are presented in the last section.

Throughout this paper, $\|\cdot\|_{2}$ denotes the usual Euclidean norm of a vector in $\mathbb{R}^{r}$, and $\|\cdot\|$ denotes the 2 -norm of a square matrix in $\mathbb{R}^{r \times r}$.

\section{Convergence of the formal series solution}

From section 4 of [16], existence solutions of (5)-(8) are guaranteed and, given $T>0$, from theorems 3.2 and 3.3 of [16], under the assumptions of the problem, there exists a constant $\delta$ such that

$$
\left\|Y_{n}(t)\right\| \leq \delta, \quad\left\|\widetilde{Y_{n}}(t)\right\| \leq \frac{\delta d}{n \pi}\left(\left\|P^{-1}(0)\right\|\|Q(0)\|^{-1}\right)^{1 / 2},
$$

for all $n \geq 1$ and $0 \leq t \leq T$, where $\delta$ depends only of the data $\|P(0)\|$, $\|P(t)\|,\left\|P^{-1}(0)\right\|,\left\|P^{-1}(t)\right\|,\|Q(0)\|,\|Q(t)\|,\left\|Q^{-1}(0)\right\|,\left\|Q^{-1}(t)\right\|$, and the size of the matrices and vectors $r$.

Under the assumptions III)-IV) and from [19, p. 71], the sine Fourier coefficients $a_{n}, b_{n}$, satisfy

$$
\left\|a_{n}\right\|_{2} \leq \frac{L_{1}}{n^{3}}, \quad\left\|b_{n}\right\|_{2} \leq \frac{L_{2}}{n^{2}}, \quad n \geq 1
$$


where

$$
L_{1}=\frac{2 d^{2}}{\pi^{3}} \int_{0}^{d}\left\|f^{(3)}\right\|_{2}(x) d x, \quad L_{2}=\frac{2 d}{\pi^{2}} \int_{0}^{d}\left\|g^{(2)}\right\|_{2}(x) d x
$$

thus

$$
\sum_{n \geq 1}\left\|Y_{n}(t)\right\|\left\|a_{n}\right\|_{2}<+\infty, \quad \sum_{n \geq 1}\left\|\tilde{Y}_{n}(t)\right\|\left\|b_{n}\right\|_{2}<+\infty
$$

see [9, pp. 38-41], and the series (3) is uniformly convergent in $D(d, T)$ and defines a continuous function that is a rigorous solution of problem (1)-(2), see theorem 4.1 of [16].

On the other hand, we can choose $n_{0}>0$ such that the error, i.e., the sum of terms in (3) for $n>n_{0}$, is smaller than an arbitrary predetermined bound. In fact, from section 5 of [16], given an arbitrary $\epsilon>0$, it suffices to take a number of terms in the series smaller than $n_{0}$, where $n_{0}$ is the first integer such that

$$
n_{0} \epsilon \geq\left\{\frac{23 \delta}{8}\left(L_{1}+\frac{d L_{2}\left\|P^{-1}(0)\right\|^{1 / 2}}{\pi\|Q(0)\|^{1 / 2}}\right)\right\}^{1 / 2}, \quad n_{0} \geq 2
$$

where $L_{i}, i=1,2$, are given by (10). Furthermore, in practice, the constants in (11) can be enlarged sometimes and the series may be truncated before $n_{0}$.

Our goal is to apply time-averaging methods based on the Magnus series expansion jointly with exponential integrators for the numerical integration of IVPs (6)-(7). Geometric integrators have shown in many cases a high performance for the numerical integration of IVPs, not only for a high numerical performance, also because they preserve some of the qualitative properties of the exact solution, see [6]. As we will see, an appropriate choice of exponential integrators allows to obtain accurate and stable solutions at a low computational cost in comparison to standard numerical integrators.

\section{Numerical approximation for the IVPs}

\subsection{The autonomous case}

Let us first consider the case in which $Q$ and $P^{-1}$ are positive definite constant matrices. In this case, the equations for $Y_{n}(t)$ and $\widetilde{Y}_{n}(t)$ have exact solution in a closed form. At this point, it is interesting to present the following results. 
Let $N, M \in \mathbb{R}^{r}$ symmetric positive definite matrices, then

$$
\begin{gathered}
\exp \left(t\left[\begin{array}{cc}
0 & M \\
-N & 0
\end{array}\right]\right) \\
=\left[\begin{array}{cc}
\cos (t \sqrt{M N}) & M(\sqrt{N M})^{-1} \sin (t \sqrt{N M}) \\
-N(\sqrt{M N})^{-1} \sin (t \sqrt{M N}) & \cos (t \sqrt{N M})
\end{array}\right],
\end{gathered}
$$

and by considering the following property for any matrix $R \in \mathbb{R}^{r \times r}$

$$
\exp ((m+1) R)=\exp (m R) \exp (R)
$$

we can check, by replacing $t$ by $m+1$ in (12) that

$$
\begin{aligned}
\cos ((m+1) S) & =\cos (m S) \cos (S)-\sin (m S) \sin (S) \\
\sin ((m+1) S) & =\sin (m S) \cos (S)+\cos (m S) \sin (S)
\end{aligned}
$$

where, in our case, we can take as the matrix $S$ either $\sqrt{M N}$ or $\sqrt{N M}$.

If we take $M=P^{-1}, N=n^{2} Q$, which are positive definite matrices, and taking into account that

$$
\Phi_{n}(T, 0)=\exp \left(T\left[\begin{array}{cc}
0 & P^{-1} \\
-n^{2} Q & 0
\end{array}\right]\right),
$$

we find that

$$
\begin{gathered}
\Phi_{n}(T, 0) \\
=\left[\begin{array}{cc}
\cos \left(n T \sqrt{P^{-1} Q}\right) & \frac{1}{n} P^{-1}\left(\sqrt{Q P^{-1}}\right)^{-1} \sin \left(n T \sqrt{Q P^{-1}}\right) \\
-n Q\left(\sqrt{P^{-1} Q}\right)^{-1} \sin \left(n T \sqrt{P^{-1} Q}\right) & \cos \left(n T \sqrt{Q P^{-1}}\right)
\end{array}\right] .
\end{gathered}
$$

To compute $\Phi_{1}(T, 0)$ requires to evaluate

$$
\cos \left(T \sqrt{P^{-1} Q}\right), \quad \cos \left(T \sqrt{Q P^{-1}}\right), \quad \sin \left(T \sqrt{P^{-1} Q}\right), \quad \sin \left(T \sqrt{Q P^{-1}}\right) .
$$

Then, the computation of $\Phi_{n}(T, 0), n>1$ can be carried out just by taking into account the recursive relations (13) and (14), which only involve a few matrix multiplications. 


\subsection{The non-autonomous case}

In this section, we consider the IVPs $(6)-(7)$, where $M(t, n)$ is given by (8). If now $\Phi_{n}\left(t, t_{0}\right)$ denotes the fundamental matrix solution of the homogeneous matrix equation

$$
\Phi_{n}^{\prime}\left(t, t_{0}\right)=M(t, n) \Phi_{n}\left(t, t_{0}\right), \quad \Phi_{n}(t, t)=I \in \mathbb{R}^{2 r \times 2 r},
$$

then, the solution of the homogeneous equations (6)-(7) can be written in the form

$$
V_{n}(t)=\Phi_{n}(t, 0) V_{n}(0), \quad W_{n}(t)=\Phi_{n}(t, 0) W_{n}(0) .
$$

Note that the dependence in $n$ is given by $\Phi_{n}$. From the initial conditions, $V_{n}(0)$ and $W_{n}(0)$ remain the same for all $n \geq 1$.

If we denote by $V_{l, n}, l=0,1, \ldots, L$, the values of $V_{n}(t)$ at a mesh $t_{0}=0$, $t_{1}=h, \ldots, t_{L}=L h$, with $h=T / L$, and $\Phi_{n, l} \equiv \Phi_{n}\left(t_{l}+h, t_{l}\right)$, then

$$
V_{l+1, n}=\Phi_{n, l} V_{l, n}, \quad l=0,1, \ldots, L-1 .
$$

At this point it is convenient to analyse the structure of the fundamental matrix solution. It is easy to prove that, for this problem, the matrix, $\Phi_{n, l}$, takes the form

$$
\Phi_{n, l}=\left[\begin{array}{cc}
a_{n, l} & \frac{1}{n} b_{n, l} \\
n c_{n, l} & d_{n, l}
\end{array}\right],
$$

where $a_{n, l}, b_{n, l}, c_{n, l}, d_{n, l}$ are functions bounded by a constant which does not depend of $n$, and $\Phi_{n, l}=I+\mathcal{O}(h)$ in the limit $h \rightarrow 0$ (or $\left.L \rightarrow \infty\right)$.

Then, for the integration over a finite time interval, $t \in[0, T]$, with $h=$ $T / L$, we have that

$$
\Phi_{n}=\prod_{l=0}^{L-1} \Phi_{n, l}=\left[\begin{array}{cc}
A & \frac{1}{n} B \\
n C & D
\end{array}\right]
$$

where $A, B, C, D$ are functions which can also be bounded by functions depending on $T, P(t), Q(t)$, but not on $n$. As a result, standard explicit methods like explicit Runge-Kutta methods are not appropriate for solving this problem because for a fixed value of $h$, the numerical solution grows polynomially with $n$ and the series solution will not converge so, one has to use implicit methods. 
The matrices $\Phi_{n, l}$ for $l=0,1, \ldots, L-1, n=1,2, \ldots, n_{0}$, have to be numerically approximated, and this can lead to exceedingly costly algorithms. If one uses the same time step, $h$, for all values of $n$, the matrices $P(t)$ and $Q(t)$ will need to be computed only once on a mesh, i.e. the same values can be used for all $n$. However, as we will see, the performance of most standard methods rapidly deteriorate with $n$. In addition, implicit methods usually need to compute the inverse of a matrix (the problem is linear) and the algorithm has to repeat this computation $L \cdot n_{0}$ times.

In the following, we show that some exponential methods have many advantages for the numerical integration of this problem. The exponential methods we consider in this work are explicit methods, but closely related to implicit methods for linear problems [6]. They show a better performance for large values of $n$ (they converge to the exact solution in the limit when $P(t), Q(t)$ are constant) and there is a recursive algorithm which allows to compute all matrices $\Phi_{n, l}, n=2,3, \ldots, n_{0}$ from $\Phi_{1, l}$.

\subsection{Second order exponential integrator}

We consider now some exponential integrators for non-autonomous linear problems in order to solve the matrix equation (15). These methods have shown to be superior, both qualitatively and quantitatively, to standard methods for solving many linear IVPs. For example, for problems with oscillatory solutions or for stiff problems, the effectiveness of these methods is far superior to traditional methods, like Runge-Kutta methods, see $[4,5,6,7,11,15]$.

We first consider in detail the following second order approximation (in the time step $h$ ) to the fundamental matrix $\Phi_{n}$

$$
\exp \left(\int_{t}^{t+h} M(t, n)\right)=\Phi_{n}(t+h, t)+\mathcal{O}\left(h^{3}\right),
$$

that corresponds to the first order approximation for most exponential methods like e.g. the Magnus, Fer or Wilcox expansions (see [2] and the references therein for details). Here, it suffices to approximate the integral by a second order symmetric rule. From the computational point of view, we found it useful to consider the trapezoidal rule

$$
\Psi_{n, l} \equiv \exp \left(\frac{h}{2}\left(M\left(t_{l}+h, n\right)+M\left(t_{l}, n\right)\right)\right)=\Phi_{n, l}+\mathcal{O}\left(h^{3}\right) .
$$


Notice that given $s>0$ and $b_{i}>0,0 \leq c_{i} \leq 1, i=1, \ldots, s$ we have that

$$
\sum_{i=1}^{s} b_{i} M\left(t+c_{i} h, n\right)=\left[\begin{array}{cc}
0 & \hat{P}^{-1}(t) \\
-\left(\frac{n \pi}{d}\right)^{2} \hat{Q}(t) & 0
\end{array}\right]
$$

where

$$
\hat{P}(t)=\left(\sum_{i=1}^{s} b_{i} P^{-1}\left(t+c_{i} h\right)\right)^{-1}, \quad \hat{Q}(t)=\left(\sum_{i=1}^{s} b_{i} Q\left(t+c_{i} h\right)\right),
$$

and are positive definite matrices which do not depend on $n$. If one relaxes the condition $b_{i}>0$ to $\sum_{i=1}^{s} b_{i}>0$ then, from the smoothness of the matrices $P(t), Q(t)$ we can find a value, $h^{*}$ such that if $h<h^{*}$ it is still guaranteed that $\hat{P}(t), \hat{Q}(t)$ are positive definite matrices. Notice that in these cases we can compute $\Psi_{n, l}$ from $\Psi_{1, l}$ using the recursive schemes (13) and (14).

Summarizing, a symmetric second order exponential integrator is given by

$$
\left.\begin{array}{c}
V_{l+1, n}=\exp \left(\frac{h}{2}\left(M\left(t_{l}+h, n\right)+M\left(t_{l}, n\right)\right)\right) V_{l, n} ; \\
V_{0, n}=\left[\begin{array}{c}
I_{r} \\
0_{r \times r}
\end{array}\right] ; l=0,1, \ldots, L-1, \quad L h=T .
\end{array}\right\}
$$

The same arguments should be applied to $W_{n}(t)$

$$
\left.\begin{array}{c}
W_{l+1, n}=\exp \left(\frac{h}{2}\left(M\left(t_{l}+h, n\right)+M\left(t_{l}, n\right)\right)\right) W_{l, n} ; \\
W_{0, n}=\left[\begin{array}{c}
0_{r \times r} \\
P(0)
\end{array}\right] ; l=0,1, \ldots, L-1, \quad L h=T .
\end{array}\right\}
$$

\subsection{High order exponential integrators. Commutator-free methods}

Magnus integrators up to very high orders can be found in the literature (see $[2,3]$ and references therein). These methods, as well as most exponential integrators for linear problems, involve the computation of the exponential of a matrix involving commutators. While this is not a serious problem for one single exponential, it is not a simple task to build a recursive algorithms to compute the the matrix solution for $n>1$ from the computation for $n=1$. For such reason, we consider more appropriate to use commutator-free 
Magnus integrators. A simple two exponential fourth-order commutator-free method for the interval $t \in\left[t_{l}, t_{l}+h\right]$ is given by [5]

$$
\Psi^{[4]} \equiv \exp \left(\frac{h}{2}\left(\beta M_{1}+\alpha M_{2}\right)\right) \exp \left(\frac{h}{2}\left(\alpha M_{1}+\beta M_{2}\right)\right)
$$

with $\alpha=\frac{1}{2}+\frac{\sqrt{3}}{3}, \beta=\frac{1}{2}-\frac{\sqrt{3}}{3}, M_{i}=M\left(t_{l}+c_{i} h\right)$, and $c_{1}=\frac{1}{2}-\frac{\sqrt{3}}{6}, c_{2}=\frac{1}{2}-\frac{\sqrt{3}}{6}$.

Notice that each exponent has the form (17) and a similar recursion as for the second order method can be used to compute the exponentials for $n>1$. Notice that $\beta<0$, but $\frac{1}{2}(\alpha+\beta)=\frac{1}{2}>0$. However, all existing commutator-free methods of order six or higher $[1,5]$ are such that at least one of the exponent has the form (17) with $\sum_{i} b_{i}<0$, and this can cause serious troubles on the existence and positivity of the matrices.

\section{On the convergence of the numerical scheme}

For the sake of clarity in the presentation, let us consider the matrix problem

$$
Z^{\prime}(t)=\widetilde{M}(t, n) Z(t)
$$

where $\widetilde{M}(t, n)$ is given by

$$
\widetilde{M}(t, n)=\left[\begin{array}{cc}
0 & p^{-1}(t) \\
-\left(\frac{n \pi}{d}\right)^{2} q(t) & 0
\end{array}\right] .
$$

Here we consider $p(t)$ and $q(t)$ are scalar functions verifying the corresponding conditions in the scalar case. Applying the numerical algorithm (16) we have

$$
Z_{l+1, n}=\exp \left(\frac{h}{2}\left(\widetilde{M}\left(t_{l}+h, n\right)+\widetilde{M}\left(t_{l}, n\right)\right)\right) Z_{l, n}
$$

Note that

$$
\frac{h}{2}\left(\widetilde{M}\left(t_{l}+h, n\right)+\widetilde{M}\left(t_{l}, n\right)\right)=\left[\begin{array}{cc}
0 & \alpha(l, h) \\
-n^{2} \beta(l, h) & 0
\end{array}\right],
$$

where

$$
\alpha(l, h)=\frac{h}{2}\left(p^{-1}\left(t_{l}+h\right)+p^{-1}\left(t_{l}\right)\right), \quad \beta(l, h)=\frac{h \pi^{2}}{2 d^{2}}\left(q\left(t_{l}+h\right)+q\left(t_{l}\right)\right) .
$$


Thus, following the same arguments shown in the subsection 3.3

$\exp \left[\begin{array}{cc}0 & \alpha(l, h) \\ -n^{2} \beta(l, h) & 0\end{array}\right]=\left[\begin{array}{cc}\cos (n \gamma(l, h)) & \frac{1}{n} \frac{1}{\rho(l, h)} \sin (n \gamma(l, h)) \\ -n \rho(l, h) \sin (n \gamma(l, h)) & \cos (n \gamma(l, h))\end{array}\right]$

where

$$
\gamma(l, h)=\sqrt{\alpha(l, h) \beta(l, h)}, \quad \rho(l, h)=\sqrt{\frac{\beta(l, h)}{\alpha(l, h)}} .
$$

In order to achieve the step $L$, the product of $L$ matrices of this type must be performed. But note that the result is a matrix with the same dependence in $n$

$$
\left[\begin{array}{cc}
\mu_{1}(n, h) & \frac{1}{n} \mu_{2}(n, h) \\
-n \mu_{3}(n, h) & \mu_{4}(n, h)
\end{array}\right],
$$

where $\mu_{i}(n, h), \quad 1 \leq i \leq 4$, are a span of bounded trigonometric functions.

Multiplying for the appropriate starting condition and taking into account (5)-(8), it follows that

$$
\begin{gathered}
y_{n, L}=\left[\begin{array}{ll}
1 & 0
\end{array}\right]\left[\begin{array}{cc}
\mu_{1}(n, h) & \frac{1}{n} \mu_{2}(n, h) \\
-n \mu_{3}(n, h) & \mu_{4}(n, h)
\end{array}\right]\left[\begin{array}{l}
1 \\
0
\end{array}\right]=\mu_{1}(n, h), \\
\widetilde{y}_{n, L}=\left[\begin{array}{ll}
1 & 0
\end{array}\right]\left[\begin{array}{cc}
\mu_{1}(n, h) & \frac{1}{n} \mu_{2}(n, h) \\
-n \mu_{3}(n, h) & \mu_{4}(n, h)
\end{array}\right]\left[\begin{array}{c}
0 \\
p(0)
\end{array}\right]=\frac{1}{n} \mu_{2}(n, h) p(0) .
\end{gathered}
$$

From (9) we have

$$
y_{n, L} a_{n}+\widetilde{y}_{n, L} b_{n} \longrightarrow \frac{1}{n^{3}}
$$

and the series

$$
\sum_{n \geq 0}\left\{y_{n, L} a_{n}+\widetilde{y}_{n, L} b_{n}\right\}
$$

is uniformly convergent.

In this way, the advantages of exponential methods are clear versus standard multi-step numerical methods for this problem. The dependence on $n$ after $L$ steps given by (20) is essential in order to ensuring the convergence of the series. For instance, if an explicit Runge-Kutta method is applied, we have

$$
Z_{L, n}=P\left(\widetilde{M}\left(t_{L}, n\right)\right) Z_{0, n}
$$


where $P\left(\widetilde{M}\left(t_{L}, n\right)\right)$ is a polynomial in $n$ of degree $L+1$. Also, for implicit Runge-Kutta methods, the relationship between the last step and the starting condition has the form

$$
Z_{L+1, n}=n F(n h) Z_{0, n}
$$

such that, if the series is truncated at $n_{0}$, it's necessary to take $h \ll 1 / n_{0}$ in order to ensure that $F(n, h)$ provides a close approximation of the solution. For more details, see [12] and references therein.

\section{Testing the numerical algorithm}

For simplicity, let us suppose that $u(x, 0)=u_{t}(x, 0)$, and then, $f(x)=$ $g(x), 0 \leq x \leq d$. In this way, the Fourier coefficients $a_{n}$ and $b_{n}$ given by (4) are equal and, from (5), we can write (3) as

$$
u(x, t)=\left[\begin{array}{ll}
I_{r} & 0_{r \times r}
\end{array}\right] \sum_{n \geq 1} \sin \left(\frac{n \pi x}{d}\right) \Psi_{n}(t) a_{n},
$$

where

$$
\Psi_{n}(t)=\left(V_{n}(t)+W_{n}(t)\right) \in \mathbb{R}^{2 r \times r}, \quad n \geq 1 .
$$

Thus, from (18)-(19), a second order exponential integrator for $\Psi_{n}(t)$ is given by

$$
\left.\begin{array}{c}
\Psi_{l+1, n}=\exp \left(\frac{h}{2}\left(M\left(t_{l}+h, n\right)+M\left(t_{l}, n\right)\right)\right) \Psi_{l, n}, \\
l=0,1, \ldots, L-1, \quad L h=T,
\end{array}\right\}
$$

where

$$
\Psi_{l, n}=V_{l, n}+W_{l, n}, \quad \Psi_{0, n}=\Psi_{0}=V_{0, n}+W_{0, n}=\left[\begin{array}{c}
I_{r} \\
P(0)
\end{array}\right]
$$

and $M\left(t_{l}, n\right)$ are the values of $M(t, h)$ given by (8), evaluated in the mesh points $t_{l}=l h$.

As an illustrative example, let us consider the scalar problem with data

$$
p(t)=3-\arctan (t), \quad q(t)=1+\arctan (t),
$$

that verify the required conditions

$$
p(t)>0, \quad q(t)>0, \quad-p^{\prime}(t)=q^{\prime}(t)=\frac{1}{1+t^{2}}>0 ; \quad t \geq 0 .
$$


Using (21) and the initial condition (22), with $p(0)$ given by (23), we can write

$$
\Psi_{l, n}=\prod_{k=0}^{l-1}\left[\exp \left(\frac{h}{2}\left(M_{l-k, n}+M_{l-k-1, n}\right)\right)\right]\left[\begin{array}{l}
1 \\
3
\end{array}\right] \text {. }
$$

Let us take $T=1$ and $h=0.01$, i.e., the interval $[0,1]$ is divided in one hundred knots such that

$$
t_{l}=l h, \quad t_{l+1}=t_{l}+h=(l+1) h, \quad t_{100}=100 h=1 .
$$

From (8) and the coefficients $p(t), q(t)$ given by (23), we have

$$
M_{l, n}=\left[\begin{array}{cc}
0 & \frac{1}{3-\arctan (l h)} \\
-\left(\frac{n \pi}{d}\right)^{2}(1+\arctan (l h)) & 0
\end{array}\right] .
$$

Thus, if we denote by

$$
\psi_{l, n}(x)=\left[\begin{array}{ll}
1 & 0
\end{array}\right] \sum_{k=1}^{n} \sin \left(\frac{k \pi x}{d}\right) \Psi_{l, k},
$$

where $\Psi_{l, k}$ is given by (24), then the approximate solution of (1) at $t=1$, is given by

$$
u(x, 1)=\sum_{n \geq 1} \psi_{100, n}(x) a_{n},
$$

where $a_{n}=b_{n}$, is given by (4).

In order to show the performance of the exponential methods considered in this work when taking large values of $n$ we consider separately both components of $\Psi_{l, n}=\left(y_{1}, y_{2}\right)$ in (22). In Figure 1 we show the error at final time, $T=1$ and constant time step, $h=1 / 100$ of both components when considering the second order Magnus integrator (22) and when considering the implicit second order trapezoidal Runge-Kutta method. The superiority of the exponential method as the value of $n$ grows is clear.

In order to check the convergence of the series (25) for $l=100$, the figure 2 shows the results for $\psi_{100, n}$, choosing $d=1$ and $x=0.3, x=0.6, x=0.9$, respectively. Note that, taking just over ten terms, the series offers stability in the results for all cases.

In order to verify the effectiveness and simplicity in the implementation of the proposed numerical method, we repeat the previous calculus for the 

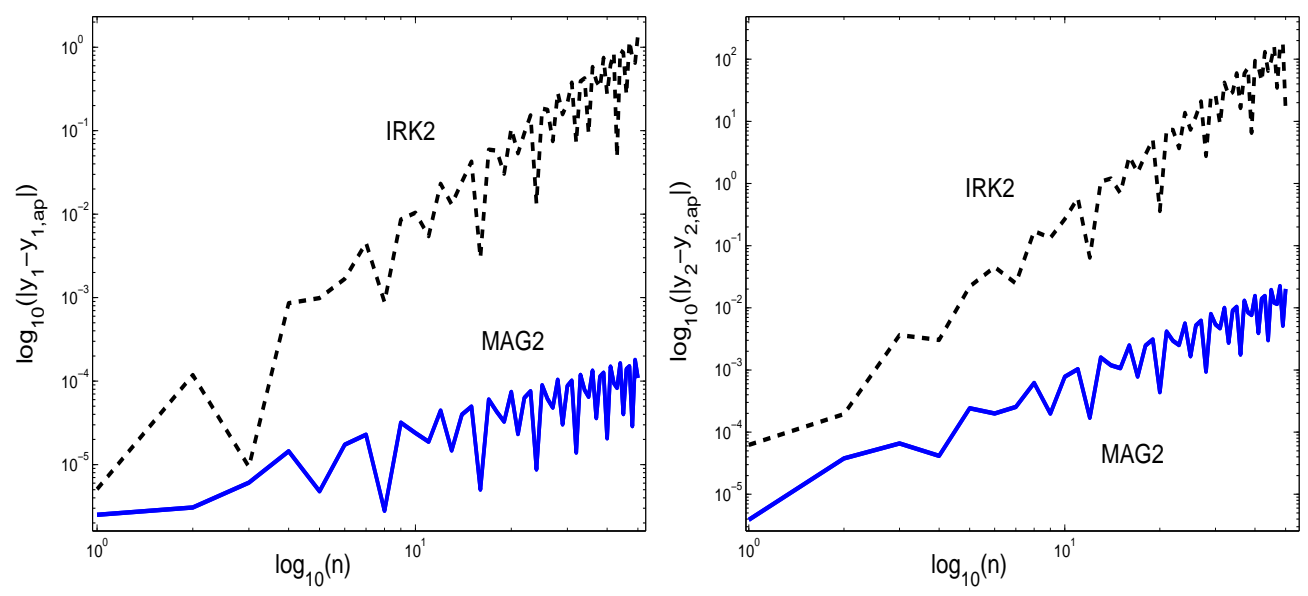

Figure 1: Error at the final time for the following methods: the implicit second order trapezoidal Runge-Kutta method and the second order Magnus integrator, both for $n=$ $1,2, \ldots, 50$.

case $d=2$ at $T=1$. Figure 3 corresponds to $\psi_{100, n}$ for values of $x$ at $0.5,1$ and 1.5. Note that for a higher spatial domain, it is necessary to take more number of terms in the series to obtain convergence. However, for larger values of $T$, the series stabilizes quickly.

In figure 4 , also for $d=2$ and taking $T=10$ with the same $h=0.01$, i.e., taking one thousand knots in the time domain $[0,10]$, we observe that is suffices to take less than five terms in the series to stabilize the results.

Finally, figure 5 shows the evolution in the spatial domain of $\psi_{l, n}(x)$ for $T=1$ i.e., taking $l=100$. Here, the number of terms in the series is taken for $n=100$ and $d=1,2$.

\section{Conclusions}

The non-autonomous matrix partial differential system (1)-(2) admits a unique solution if conditions I)-IV) are satisfied. In this case, (3) is an exact formal series solution that present two important drawbacks. Firstly, (3) is an infinite series and, secondly, is necessary to solve non-autonomous IVPs, but the exact solution of these problems is not computable, in general, in an analytic way. This motivates the search of alternatives which provide approximate solutions. 
From (11), for a given admissible error $\epsilon>0$, the term from which the infinite series may be truncated so that the terms that are removed have an influence of an amount less than $\epsilon$, is given in function of the data. But, as indicated in section 2, the constants that appears in (11) can be enlarged so that the series may converge taking less terms therein.

In this sense, it is important to choose an appropriate algorithm in order to solve numerically these IVPs. We present a symmetric second order exponential integrator which show a high performance for these problems. These method leads to expression (24)-(25), that it's easy to implement and not have a high computational cost. Here, is important to recall that, as seen in section 3 , once evaluated for $n=1$, the proposed method admits a recursive scheme to find the solutions for $n>1$ and is proved that, from the qualitative properties which has, at successive iterations the error grows similarly as does the exact solution and hence the convergence of the series is unaffected. This does not happen when other standard numerical methods are used. The numerical solutions provided verify that the difference with the theoretical exact solution differs in a quantity less than $h^{3}$, where $h$ is the measure of meshing which divides the time domain. For better accuracy we can make a finest mesh. Also, higher order exponential methods can be used, see [5], but from the computational point of view, we found that high-order methods for the numerical integration can lead into most costly and involved schemes, see section 4 of [6].

The numerical experiments from the previous section confirm the effectiveness of the proposed algorithm, showing succeed results without excessive computational cost. Efficient algorithms to compute the matrix exponential or its action on a vector are required, see appendix $\mathrm{B}$ of $[6]$, or $[14,18]$.

\section{Acknowledgements}

The work of S. Blanes has been partially supported by Ministerio de Ciencia e Innovación (Spain) under project MTM2010-18246-C03. The work of E. Ponsoda has also been partially supported by the Universitat Politècnica de València under project 2087.

[1] Alvermannand A. \& Fehske H. (2011) High-order commutator-free exponential time-propagation of driven quantum systems. J. Comput. Phys., 230, 5930-5956. 
[2] Blanes, S., Casas, F., Oteo, J. A. \& Ros, J., (2009) The Magnus expansion and some of its applications. Physics Reports, 470, 151-238.

[3] Blanes, S., Casas, F. \& Ros, J. (2002) High order optimized geometric integrators for linear differential equations. BIT, 42, 262-284.

[4] Blanes, S., Jódar, L. \& Ponsoda, E., (2000) Approximate solutions with a priori error bounds for continuous coefficient matrix Riccati equations. Math. Comp. Mod., 31, 1-15.

[5] Blanes, S. \& Moan, P. C., (2006) Fourth- and sixth-order commutator-free Magnus integrators for linear and non-linear dynamical systems. Appl. Numer. Math., 56, 1519-1537.

[6] Blanes, S. \& Ponsoda, E., (2012) Time-averaging and exponential integrators for non-homogeneous linear IVPs and BVPs. Appl. Num. Math., 62, 875-894.

[7] Celledoni, E. \& Iserles, A., (2000) Approximating the exponential from a Lie algebra to a Lie group. Math. Comp., 69, 1457-1480.

[8] Dibben, D. C. \& Metaxas, R., (1996) Time domain finite element analyis of multimode microwave applicators. IEEE Trans. Magn., 32, 942-945.

[9] Folland, G. B., (1992) Fourier analysis and applications. Wadworth and Brooks. Pacific Grove. C.A.

[10] Harvey, A. F., (1963) Microwave engineering. Academic Press, New York.

[11] Iserles, A., Munthe-KaAs, H. Z., Nørsett, S. P. \& Zanna, A., (2000) Lie group methods. Acta Numerica, 9, 215-365.

[12] IsErLes, A., (2004) On the method of Neumann series for highly oscillatory equations. BIT Numerical Mathematics, 44, 473-488.

[13] Metaxas, A. C. \& Meredith, R. J., (1988) Industrial microwave heating. Peter Peregrinus Ltd., London. 
[14] Moler, C. B. \& Van Loan, C. F., (2003) Nineteen dubious ways to compute the exponential of a matrix, twenty-five years later. SIAM Rev. 45, 3-49.

[15] Ponsoda, E. \& Castaño, J. I., (1999) Approximate solutions and error bounds for matrix differential equations using Fer's factorization. J. Appl. Sci. Comp., 5, 69-79.

[16] Ponsoda, E. \& Jódar, L., (1999) Numerical solutions with a priori error bounds for coupled self-adjoint time dependent partial differential systems. Math. Comp. Mod., 29, 1-18.

[17] Roussy, G. \& Pearcy, J. A., (1995) Foundations and industrial applications of microwaves and radio frequency fields . John Wiley, New York.

[18] SiDje, R. B., (1998) Expokit: A software package for computing matrix exponentials. ACM Trans. Math. Software, 24, 130-156.

[19] Zygmund, A., (2002) Trigonometric series. Cambridge University Press. Cambridge, UK.
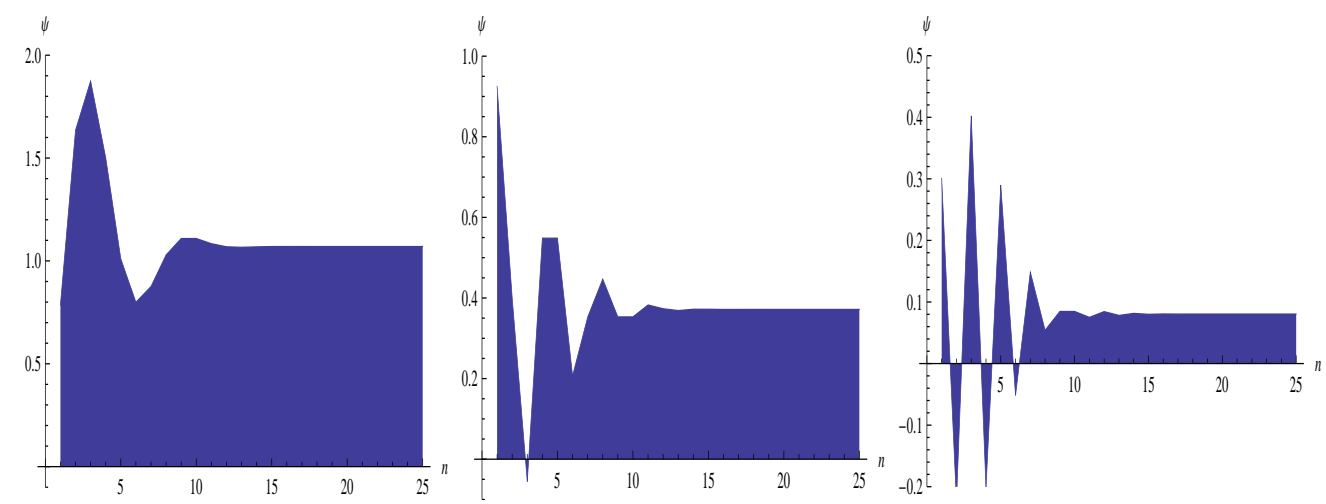

Figure 2: $\psi_{100, n}(x)$ at $x=0.3,0.6$ and 0.9 , with $d=1$. Here, $n$ is the number of terms taken in the series (25). 


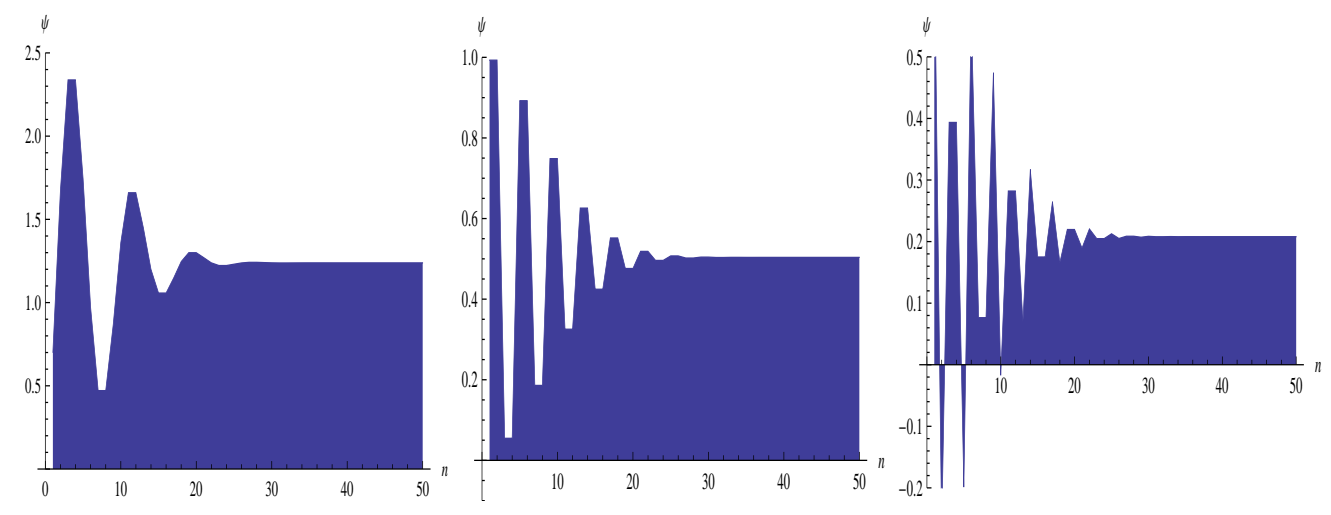

Figure 3: $\psi_{100, n}(x)$ at $x=0.5,1$ and 1.5, with $d=2$. Here, $n$ is the number of terms taken in the series (25).

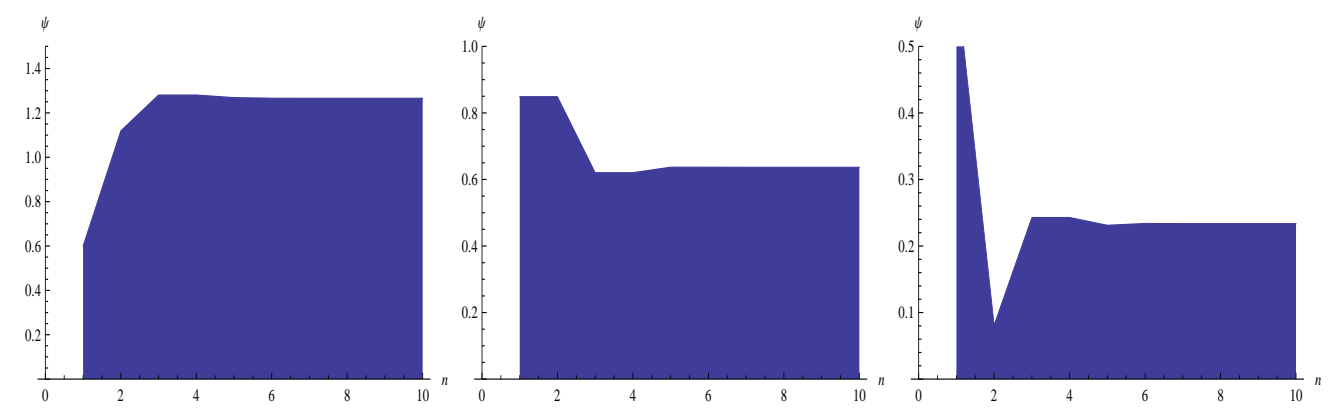

Figure 4: $\psi_{1000, n}(x)$ at $x=0.5,1$ and 1.5, with $d=2$. Here, $n$ is the number of terms taken in the series (25). 


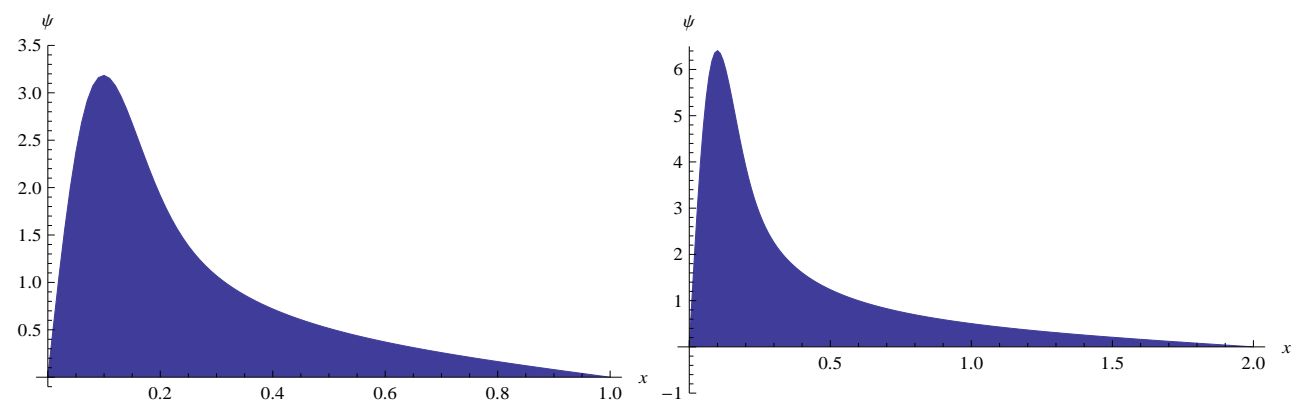

Figure 5: Left. $\psi_{100,100}(x)$, i.e, the approximation of the function $\psi(x)$ at $T=1$, taking one hundred terms in the series (25) and evaluate also in one hundred points of the spatial domain $[0,1]$. Right. The same for the spatial domain $[0,2]$. 